Published in final edited form as:

Neuropsychologia. 2010 August ; 48(10): 3019-3025. doi:10.1016/j.neuropsychologia.2010.06.010.

\title{
Neural Correlates of Familiarity-Based Associative Retrieval
}

\author{
Jaclyn Hennessey Ford ${ }^{1}$, Mieke Verfaellie 2,3 , and K.S. Giovanello ${ }^{1,4}$ \\ ${ }^{1}$ Department of Psychology, UNC-CH, Chapel Hill, NC, USA \\ ${ }^{2}$ Department of Veterans Affairs, Boston, MA, USA \\ ${ }^{3}$ Boston University School of Medicine, Boston, MA, USA \\ ${ }^{4}$ Biomedical Research Imaging Center, UNC-CH, Chapel Hill, NC, USA
}

\section{Abstract}

The current study compared the neural correlates of associative retrieval of compound (unitized) stimuli and unrelated (non-unitized) stimuli. Although associative recognition was nearly identical for compounds and unrelated pairs, accurate recognition of these different pair types was associated with activation in distinct regions within the medial temporal lobe (MTL). Recognition of previously presented compound words was associated with left perirhinal activity, whereas recognition of unrelated word pairs was associated with activity in left hippocampus. These results provide evidence that perirhinal cortex mediates familiarity-based associative memory of stimuli unitized at encoding, while the hippocampus is required for recollection-based associative memory.

\section{Keywords}

associative memory; recollection; familiarity; hippocampus; perirhinal cortex; medial temporal lobe; unitization

\section{Introduction}

Accurate recognition of a previously encountered stimulus is typically thought to rely on two mnemonic processes, recollection and familiarity. Whereas recollection provides the basis for remembering the occurrence of a stimulus and its associated contextual details, familiarity refers to an acontextual sense of awareness that arises from prior exposure to a stimulus (Mandler, 1980). Recollection and familiarity are generally thought to be supported by medial temporal lobe (MTL) structures. However, a debate centers on whether these mnemonic processes are mediated by the same or distinct MTL subregions, as well as the encoding conditions that promote the differential contribution of MTL subregions to recognition performance.

One theoretical view asserts that the entire MTL (the hippocampus plus the entorhinal, perirhinal, and parahippocampal cortices) is essential to accurate recognition memory, with

(C) 2010 Elsevier Ltd. All rights reserved.

Address correspondence to: Jaclyn Hennessey Ford, Department of Psychology, University of North Carolina at Chapel Hill, Davie Hall, C.B. \#3270, Chapel Hill, NC 27599.

Publisher's Disclaimer: This is a PDF file of an unedited manuscript that has been accepted for publication. As a service to our customers we are providing this early version of the manuscript. The manuscript will undergo copyediting, typesetting, and review of the resulting proof before it is published in its final citable form. Please note that during the production process errors may be discovered which could affect the content, and all legal disclaimers that apply to the journal pertain. 
no differential contribution of MTL substructures to recollection-based or familiarity-based processing. Support for this view comes from neuropsychological studies showing equivalent decrements in measures of recollection and familiarity whether lesions are restricted to the hippocampus or encompass the hippocampus plus parahippocampal cortex (Kirwan, Wixted, \& Squire, 2010; Stark, Bayley, and Squire, 2002; Stark \& Squire, 2003; Wais, Wixted, Hopkins, \& Squire, 2006). Additionally, findings from functional magnetic resonance imaging (fMRI) studies have demonstrated equivalent hippocampal activity when performance is thought to be mediated by recollection or familiarity (Kirwan and Stark, 2004; Stark \& Squire, 2001; Wais, Squire, \& Wixted, 2009; Wais, 2010).

A competing theoretical view suggests that recollection is critically dependent on the hippocampus, while familiarity depends on the adjacent MTL cortex (the entorhinal, perirhinal, and parahippocampal cortices). Findings from patients with hippocampal (Aggleton, Vann, Denby, Dix, Mayes, Roberts, Yonelinas, 2005; Giovanello, Keane, \& Verfaellie, 2006; Giovanello, Verfaellie, \& Keane, 2003; Turriziani, Fadda, Caltigirone, \& Carlesimo, 2004; Turriziani, Serra, Fadda, Caltigirone, \& Carlesimo, 2008) and perirhinal lesions (Bowles, Crupi, Mirsattari, Pigott, Parrent, Pruessner, Yonelinas, \& Köhler, 2007), as well as results from functional magnetic resonance imaging (fMRI) investigations (Davachi, Mitchell, \& Wagner, 2003; Giovanello, Schnyer, Verfaellie, 2004; Giovanello, Schnyer, \& Verfaellie, 2009; Henke, Buck, Weber, \& Weiser, 1997; Staresina \& Davachi, 2008; Yonelinas, Hopfinger, Buonocore, Kroll, \& Baynes, 2001), support this proposed dichotomy within the MTL.

Within this view of MTL organization, however, various models have been proposed. For example, Norman and O'Reilly's (2003) model depicts the perirhinal and the parahippocampal cortex as an intermediary between neocortex and the hippocampus (although no distinct processing roles are assigned to the perirhinal and the parahippocampal cortex). Fernandez and Tendolkar (2006) describe perirhinal cortex as the "gate-keeper" to declarative memory, having a specific role in directing encoding resources toward less familiar stimuli. Mayes and colleagues (2007) argue that the hippocampus is essential for recollection, whereas the perirhinal cortex is important for familiarity (with no specified role for parahippocampal cortex). Finally, Davachi (2006) has proposed that perirhinal cortex mediates the specific visual and conceptual features of objects, whereas parahippocampal cortex supports coding of spatial context. Information from these regions is then sent to the hippocampus where domain-general relational representations are formed.

More recently, the Binding of Item and Context (BIC) model, originally proposed by Eichenbaum, Yonelinas, and Ranganath (2007), and then refined by Diana, Yonelinas, and Ranganath (2007), suggests that there is no simple matching between MTL regions and recollection and familiarity. Rather, the involvement of different MTL regions in these processes depends not only on the mnemonic demands of the task, but also on the type of information to be encoded and retrieved. Specifically, the BIC model states that perirhinal cortex subserves familiarity-based processing, whereas the parahippocampal cortex and hippocampus both mediate recollection through their role in processing of contextual information and item-context binding, respectively. Support for the differential roles of perirhinal cortex and hippocampus comes from studies showing neural activity in perirhinal cortex during item recognition, a task that can be supported by familiarity, and neural activity in hippocampus during associative recognition, a task that typically requires conscious recollection (e.g. Hockley and Consoli, 1999). A key prediction of the BIC model, however, is that when associative recognition can be based on familiarity, performance should be mediated by perirhinal cortex. Prior studies have demonstrated that associative recognition can be based on familiarity when items are unitized at encoding unitized (Quamme, Yonelinas, \& Norman, 2007; Giovanello, Keane, \& Verfaellie, 2006). As such, 
neural mediation of associative memory may be different for unitized and non-unitized stimuli.

Indeed, prior neuropsychological studies have shown that individuals who demonstrate poor performance on typical tests of associative memory (i.e. older adults and amnesic patients with hippocampal damage) perform at significantly higher levels when provided with instructions to encode items as a unit (Quamme, Yonelinas, \& Norman, 2007) or when paired stimuli formed a pre-existing association (Giovanello, Keane, \& Verfaellie, 2006). Analysis of ROC curves (Quamme, Yonelinas, \& Norman, 2007) and Remember/Know data (Giovanello, Keane, \& Verfaellie, 2006) suggested that this enhancement was mediated by familiarity. Other studies in normal cognition also provide evidence for the operation of familiarity when items have been unitized (Diana, Yonelinas, \& Ranganath, 2008; Yonelinas, Kroll, Dobbin, \& Soltani, 1999). Finally, neuroimaging data point to a role for perirhinal cortex in the encoding of unitized stimuli. For example, in a recent fMRI study (Haskins, Yonelinas, Quamme, \& Ranganath, 2008), participants encoded novel word pairs either by reading a sentence that included the two words (non-unitized encoding) or by reading a definition for a new compound word made up of these words (unitized encoding). Neural activity was identified in the perirhinal cortex during unitized encoding of word pairs relative to non-unitized encoding. Importantly, activity in this same perirhinal locus predicted subsequent levels of familiarity-based associative recognition at retrieval (Haskins et al., 2008).

The central goal of the current study was to extend Haskins and colleagues' (2008) findings by examining whether or not the same regions engaged during encoding of unitized information (i.e., perirhinal cortex) would be re-engaged during retrieval, providing evidence for the role of perirhinal cortex in associative familiarity. We operationalized unitization as a pre-existing association between stimuli, rather than an instructional manipulation at encoding (as in Haskins et al, 2008), thereby aiming to generalize the conditions under which unitization may support familiarity-based associative memory. Finally, as a secondary goal, we investigated the contribution of the hippocampus to retrieval of non-unitized and unitized stimuli. In light of our previous finding in amnesic patients (using analogous stimuli, Giovanello et al., 2006), we hypothesized that greater hippocampal activity would be observed during retrieval of non-unitized, relative to unitized, associations.

To this end, we utilized blood oxygen level dependent (BOLD) functional MRI to identify neural activity during retrieval of unitized and non-unitized pairs, focusing on the relative contribution of different MTL structures to associative recognition of these pair types. Nonunitized word pairs consisted of two unrelated words (e.g., "dog" and "couch") that participants used to form a single sentence at encoding (e.g., "The dog sat on the couch"). Unitized word pairs consisted of the two components of a compound word (e.g. "fire" and "man") that were used in their unitized form at encoding (e.g. "The fireman carried an ax."). During retrieval, participants distinguished between old pairs and "recombined" pairs consisting of two elements that had been studied, but not together. Similar to the original pairs from which they were derived, recombined pairs were either unrelated pairs or compound stimuli.

Compound words were chosen for the unitized condition in this study because they are unique, having both integrative features (e.g., idiosyncratic meanings not completely predicted by the meanings of the constituent words), as well as some preservation of the distinct constituent components (e.g., as evidenced by increased false alarm rates to novel compounds consisting of studied components not previously seen together; e.g. Giovanello, Keane, and Verfaellie, 2006). Additionally, in a prior study we observed that familiarity 
made a greater contribution to recognition of compounds than to unrelated stimuli in healthy individuals (Giovanello, Keane, and Verfaellie, 2006). As such, in the current study we employed compound words, as they are uniquely suited to create conditions in which familiarity contributes to associative recognition.

\section{Methods}

\subsection{Participants}

Eighteen healthy young adults between the ages of 19 and $27(M=23.1 ; S D=3.7 ; 12$ female) participated in the current study. Three additional participants, who failed to follow task instructions and whose data were outliers relative to the rest of the group, were excluded from the analysis. Participants were recruited using flyers posted on the UNC campus and were paid for their participation. Participants were all right-handed native English speakers without a history of psychiatric illness or neurological disorder. Before participating in the study, participants gave written informed consent in accord with the requirements of the Institutional Review Board at the University of North Carolina at Chapel Hill.

\section{2 Materials}

Stimuli consisted of unrelated word pairs and compound words. The unrelated and compound stimuli each consisted of 64 triplets that were divided into two sets for purposes of counterbalancing across "intact" and "recombined" conditions. A "triplet" was comprised of two compound words (e.g., motorcycle and boxcar) or two unrelated word pairs (e.g., poker-curl and pact-coffee) that were recombined into a third word or pair (e.g., motorcar or poker-coffee) that served as the stimulus that appeared in the test phase (unrelated word written frequency $=13.62$; compound word frequency $=3.4$ ).

Separate study and test lists were used for the different types of stimuli to allow for different strategies participants may use for the two sets of stimuli to be optimally engaged, and as such, to maximize our ability to detect differential contributions of recollection and familiarity to the unitized and non-unitized conditions, respectively. Both study lists consisted of 32 "intact" stimuli (e.g. motorcar or poker-coffee), which appeared in the same combination in the test phase, and 64 stimuli (e.g. motorcycle and boxcar or poker-curl and pact-coffee) whose components were recombined to form 32 "recombined" stimuli in the test phase. Assignment of triplets to the "intact" and "recombined" conditions was counterbalanced across participants for both word types.

\subsection{Behavioral Procedure}

Participants studied compound words and unrelated words pairs in two consecutive study tasks. The order of study sessions was counterbalanced across participants. Following a fifteen-minute delay, participants took part in two test sessions that corresponded to the two study lists.

During the study phase, participants viewed compound words or unrelated word pairs presented in black type on a white computer screen. Unrelated words and compound word components were separated by a blank space on the screen. For each word pair, participants created a simple sentence including the compound word or the words in the unrelated pair. For example, if a participant was presented with the words "dog" and "couch," they would produce a short sentence such as, "The dog sat on the couch." For the compound condition (i.e. "mail" and "box" separated by a space), participants generated a sentence with the words combined into the compound (i.e., "The man went to the mailbox"). Participants indicated via button press when they had successfully generated a sentence. The study phase was self-paced, progressing to the next stimulus following the button press. 
Once participants had completed the study phase, they were placed in the scanner to participate in the test phase. Imaging data were acquired in two scanner runs - one for compound stimuli and one for unrelated word pairs. Order of stimulus type was counterbalanced across participants. Stimuli were back-projected onto a white screen behind the participants, who viewed the stimuli using a mirror fixed to the head coil. During the test phase, participants viewed intact and recombined pairs and were asked whether the two words had been seen together previously. Participants made yes/no judgments for 64 pairs in each run. Participants were instructed to select "yes" if the two words had appeared together at study and to respond "no" if they had not. Each pair was presented for three seconds, a duration that pilot studies demonstrated to be sufficient for a judgment. A simple control task was included that asked participants to identify the spatial location (left or right) of a row of ampersands on the computer screen. The length of the control task randomly varied from three to nine seconds to create temporal jitter of the events.

\subsection{Data Acquisition}

Magnetic resonance images were acquired using a Siemens Trio 3-T scanner. Participants' heads were held in place using cushions and a headrest. First, $1601 \mathrm{~mm}$ structural images ( $\mathrm{TR}=1750 \mathrm{~ms}, \mathrm{TE}=4.38 \mathrm{~ms}$ ) were collected. Following the $\mathrm{T} 1 \mathrm{scan}$, two functional scans were performed during the behavioral testing sessions for the compound and unrelated word lists. In each scan, fifty slices ( $3 \mathrm{~mm}$ thick, $\mathrm{TR}=3 \mathrm{~s}, \mathrm{TE}=23 \mathrm{~ms}$ ) were acquired at an angle parallel to the long axis of the hippocampus, identified during T1 scan. All behavioral responses were recorded using an MR-compatible response box.

\subsection{Data Analysis}

Images were preprocessed and analyzed using SPM8 software implemented in MATLAB (Statistical Parametric Mapping; Wellcome Department of Cognitive Neurology, London, UK). Images were re-oriented, slice-time corrected, realigned and unwarped, normalized and smoothed using a Gaussian $8 \mathrm{~mm}$ kernel. Images corresponding to correct test trials only (hits and correct rejections for compounds and unrelated word pairs) were analyzed.

For each participant, on a voxel-by-voxel basis, an event-related analysis was first conducted in which all instances of a particular event type (i.e. unrelated intact pair hit, unrelated recombined pair correct rejection, compound intact pair hit, compound recombined pair correct rejection) were modeled through the convolution with a canonical hemodynamic response function. Effects for each event type were estimated using a subjectspecific, fixed effects model. Importantly, the neural correlates of associative recognition were measured by contrasting activity during the intact pair condition to activity during the recombined pair condition for each stimulus type (i.e. unrelated and compound pairs). These data were then entered into a second order, random-effects analysis to assess activity for these comparisons across participants. Due to our a priori hypotheses regarding the contribution of MTL structures to associative memory, as well as the extensive literature implicating the MTL in memory processing, regions consisting of at least ten contiguous voxels that exceeded the threshold of $p<0.005$ (two-tailed) were considered reliable (see also Lieberman and Cunnigham, 2009 for an argument for balancing type I and type II errors in neuroimaging research).

A conjunction analysis then examined the neural regions commonly activated for the associative recognition measure under both compound and unrelated stimulus conditions. To do so, an image mask was created for the associative recognition measure of the compound condition (i.e. compound intact pair hit greater than compound recombined pair correct rejection) at a p-value of $p<.01$. This mask was then applied to the associative recognition 
measure for the unrelated condition (i.e. unrelated pair intact hit greater than unrelated pair recombined correct rejection), which had been set at $p<.01$.

To assess neural activity differentially elicited for the compound relative to the unrelated stimuli, we conducted paired t-tests comparing the associative recognition measure for each stimulus type. More specifically, the first t-test assessed neural regions responding to compound stimuli (i.e. intact greater than recombined) to a greater extent than unrelated stimuli (i.e. intact greater than recombined). Conversely, the second t-test examined neural regions responding to unrelated greater than compound stimuli.

Finally, based on the regions identified in the paired t-tests, region of interest (ROI) analyses were conducted using Marsbar (http://marsbar.sourceforge.net; Brett, Anton, Valabregue, and Poline, 2002) to identify the contribution of each stimulus condition (i.e. unrelated intact pair hit, unrelated recombined pair correct rejection, compound intact pair hit, compound recombined pair correct rejection) to neural responses within MTL regions. Activity within these ROIs was then projected onto an MRIcron template (http://www.cabiatl.com/mricro/mricron/index.html). All activations are presented in neurological coordinates (i.e., activity in the right hemisphere is presented on the right side of the brain image). Voxel coordinates are reported in Montreal Neurological Institute (MNI) coordinates and reflect the most significant voxel within the cluster.

\section{Results}

\subsection{Behavioral Data}

Associative memory accuracy in this study was measured by subtracting the false alarm rate (incorrect "together previously" response to a recombined pair) from the hit rate (correct "together previously" response to an intact pair) for each stimulus condition (see Table 1). Participants' associative memory rates in the two word pair conditions were not significantly different from one another, $(M=.62 ; S E=.04)$ and $(M=.60 ; S E=.04)$ for unrelated word pairs and compounds, respectively; $(t=-.534, p>1)$. Hit rates to unrelated word pairs $(M=.77$; $S E=.03)$ did not differ from hit rates to compounds $(M=.81 ; S E=.03 ; t=1.198, p>1)$. Additionally, false alarm rates to unrelated word pairs $(M=.15 ; S E=.02)$ did not differ statistically from false alarm rates to compounds $(M=.21 ; S E=.03 ; t=2.035, p=.06)$.

Average response times were calculated for correct responses for each memory condition (see Table 1). For compound word pairs, participants took an average of 1.27s $(S E=.03)$ to respond to intact pairs (hits) and $1.45 \mathrm{~s}(S E=.05)$ to respond to recombined pairs (correct rejections). Averages for the unrelated word pair condition were $1.51 \mathrm{~s}(S E=.04)$ and $1.79 \mathrm{~s}$ $(S E=.05)$ for intact and recombined pairs, respectively. Contrasts revealed that participants were significantly slower responding to unrelated word pairs compared to compound word pairs $(F(1,19)=130.5, p<.001)$ and recombined pairs compared to intact pairs $(F(1,19)=52.6$, $p<.001)$. The difference between intact and recombined pairs was not significantly different between the two word pair conditions $(F(1,19=3.49, p=.08)$.

\subsection{Imaging Data}

To examine neural regions mediating associative memory recognition for both unrelated word pairs and compound words, a conjunction analysis was performed (see Methods section for description). This analysis revealed activity in left parietal cortex $(-56,-50,40)$ only. To determine whether any MTL regions were common to associative memory recognition for both unrelated and compound stimuli, we lowered the threshold to $\mathrm{p}<.05$ for each contrast. Table 2 shows regions commonly active for the two conditions with a t-value > 3.0. Here, no MTL regions were observed. 
Next, paired t-tests examined activity that was greater during retrieval of either unrelated or compound stimulus conditions (see Methods for details). Specifically, we examined regions that were more active in the intact condition relative to the recombined condition (i.e., the measure of associative memory) for both stimulus types. An examination of associative recognition related activity for compound stimuli greater than unrelated stimuli identified activity in right insula, right cuneus, right lingual gryrus, bilateral anterior cingulate, right inferior parietal lobule, right middle occipital gyrus, left perirhinal, left superior lobule, and right precuneus (see Figure 1 and Table 3). The observation of activity in left perirhinal cortex during retrieval of compound stimuli is in line with the primary hypothesis that retrieval of unitized associations can be supported by perirhinal cortex.

To determine the nature by which each experimental condition contributed to activity in perirhinal cortex, we conducted a follow-up ROI analysis of left perirhinal cortex. We observed deactivations in this region for the compound intact condition (-.03) and activations in this region for the compound recombined condition (.06; See Figure 1). Additionally, we observed minimal signal changes in this region for the unrelated intact (-. 003) and unrelated recombined (.018) conditions. Importantly, the difference in neural activity between the intact and recombined experimental conditions was greater in the compound relative to the unrelated stimuli, suggesting a role for this region is familiaritybased associative recognition.

Next, we examined regions that were more active in the intact condition relative to the recombined condition (i.e., the measure of associative memory) for unrelated stimuli. Table 4 lists all regions identified in the paired t-test examining unrelated "intact > recombined" greater than compound "intact > recombined". For this contrast we observed bilateral middle temporal gyrus, right inferior temporal gyrus, right insula, bilateral superior frontal gyrus, right middle frontal gyrus, left medial frontal gyrus, left superior temporal, left superior parietal lobe, bilateral anterior cingulate, left inferior frontal gyrus. Of note, no MTL regions were identified by this contrast. Previous research in our lab (Giovanello et al, 2004; Giovanello et al., 2009) has reported hippocampal activity during retrieval of novel associations between unrelated words, leading to an a priori hypothesis that hippocampal activity would be observed in this contrast. Consistent with these previous findings, a less conservative threshold of $p<.05(\mathrm{k}=30)$ revealed activation in left hippocampus (see Figure 2). A region of interest analysis of left hippocampus revealed activations for all experimental conditions (Compound Intact $=.02$; Compound recombined $=.02$; Unrelated Intact $=.08$; Unrelated Recombined $=.02$ ) with the unrelated intact pair condition making the greatest contribution to activity in this region. Such findings are inline with several reports documenting the role of left hippocampus in retrieval of novel associations (e.g., Giovanello, Schnyer, Verfaellie, 2004;Giovanello, Schnyer, \& Verfaellie, 2009; Yonelinas, Hopfinger, Buonocore, Kroll, \& Baynes, 2001).

\section{Discussion}

In the current study, we compared neural activity during associative retrieval of compound (unitized) stimuli and unrelated (non-unitized) stimuli. Although associative recognition was nearly identical for compounds and unrelated pairs, accurate recognition of these different pair types was associated with distinct regions within the MTL. Retrieval of compound associations preferentially engaged left perirhinal cortex, a finding that we interpret as evidence for the role of perirhinal cortex in associative familiarity. Retrieval of unrelated associations preferentially engaged left hippocampus, providing further support for the role of the hippocampus in recollection-based associative recognition. Such findings are difficult to reconcile with unitary process models of recognition memory postulating differential activity in MTL subregions based upon the relative strength (strong versus weak) of a 
memory, as recognition performance in the current study was matched between compound and unrelated stimulus conditions.

The observation of perirhinal activity during retrieval of unitized, relative to non-unitized, associations extends the findings of Haskins and colleagues (2008) by demonstrating retrieval-related functional activity in the same region (i.e., perirhinal cortex) in which, as encoding-related activity has been found to be predictive of subsequent recognition of unitized associations. Such findings suggest that the mnemonic processes engaged during encoding of unitized associations are recapitulated during retrieval of such associations, and are consistent with other lines of research in which the same neural structure has been implicated during both the encoding and subsequent retrieval of mnemonic information. For example, Johnson and Rugg (2007) recently demonstrated a content-specific relationship between encoding- and retrieval-related neural activity, whereby neural activity for remembered words overlapped with neural activity for encoded words appearing in scenes (i.e., left occipital cortex and anterior fusiform gyrus) or sentences (i.e., ventromedial frontal cortex). These findings were taken as strong support for the "reinstatement hypothesis" of episodic retrieval.

It should be noted, however, that the perirhinal activity observed by Haskins et al., (2008) was characterized by activations, whereas the perirhinal activity observed in the current study was characterized by deactivations. Such deactivations in neural activity in perirhinal cortex have been observed previously during recognition tasks, and have been attributed to re-processing of familiar information (Daselaar, Fleck, and Cabeza, 2006; Eichenbaum, Yonelinas, and Ranganath, 2007; Montaldi, Spencer, Roberts, and Mayes, 2006). In our study, neural activity decreased for compound stimuli in the "intact" condition relative to the "recombined" condition, presumably because intact compound stimuli possess familiarity for the entire stimulus (i.e., unitization) as well as familiarity for the component parts. No such difference was observed for the unrelated stimuli, suggesting a minimal contribution of these conditions to activity in perirhinal cortex.

Previous studies in our laboratory examining the functional-neuroanatomical correlates of associative recognition of unrelated words pairs have shown that left hippocampal activity is greater during correct endorsement of "intact" unrelated stimuli than during correct rejection of "recombined" stimuli, thereby demonstrating that role of this region in processing relational information (Giovanello et al., 2004; 2009). In the current study, we replicated this finding by demonstrating greater activity in left hippocampus during retrieval of "intact" relative to "recombined" unrelated stimuli ${ }^{1}$. Additionally, activity in left hippocampus was characterized by activations (as opposed to deactivations) for all stimulus conditions, and notably, activity for unrelated intact pairs was greater than activity for the other conditions. These findings point to a role for the hippocampus in associative recognition of non-unitized stimuli.

Given the differential contributions of perirhinal cortex and hippocampus to memory for different types of stimuli, it is important to consider the specific mnemonic processes that may be operating in these stimulus conditions, and additionally, whether these processes differentially contribute to accurate recognition performance. Although associative recognition for both compound and unrelated words pairs likely depends on both recollection and familiarity, prior studies using ROC curves (Quamme, Yonelinas, \& Norman, 2007) and Remember/Know data (Giovanello, Keane, \& Verfaellie, 2006) have

\footnotetext{
${ }^{1}$ To reveal hippocampal activity in the paired t-test comparing unrelated and compound pairs, a more liberal threshold of $p<.05$ was applied. As always, caution must be taken when interpreting findings at a lowered threshold. However, these data coincide with numerous previous findings, supporting their validity.
} 
demonstrated a greater contribution of familiarity to the endorsement of previously studied compound (i.e., intact stimuli) relative to unrelated stimulus pairs; while endorsement of previously studied unrelated word pairs has been shown to rely more heavily on recollection (Hockley and Consoli, 1999).

Additionally, the question arises as to the processes that allow for correct rejection of recombined pairs. One process that has been proposed to operate during recognition memory is recollection rejection (Brainerd and Reyna, 2002; Brainerd, Wright, and Reyna, 2001) or recall-to-reject (Rotello and Height, 1999, 2000). The contribution of recollection rejection to associative recognition of unrelated stimuli is well documented (Rotello and Heit, 2000; Rotello, Macmillan, \& Van Tassel, 2000). Indeed, robust evidence for recall-to-reject processing in associative recognition has been observed for word pairs, as well as listdiscrimination judgments (Rotello and Heit, 2000). Given the nature of the stimuli used in the current study (i.e., word pairs), as well as the task (i.e., associative recognition) it is highly likely that a recall-to-reject strategy contributed to participants' performance in the unrelated stimulus condition. Thus, hippocampal activity associated with accurate recognition of unrelated word pairs may reflect both recollection of target pairs and rejection of recombined pairs, through a recall-to-reject process.

Recognition memory studies of compound stimuli suggest that recollection rejection has a limited effect in successfully recognizing such stimuli (Jones and Jacoby, 2001; Jones, Jacoby, \& Gellis, 2001; Wong and Rotello, 2010), except under specific circumstances, such as when participants are presented with multiple repetitions of studied items or "warned" about the deceptive nature of the recombined stimuli (Lampinen, Odegard, and Neuschatz, 2004). Since neither of these conditions was present in the current study, recollection rejection likely had minimal impact on the ability to reject recombined stimuli. Thus, perirhinal activity associated with accurate recognition of compound pairs can best be understood in terms of the contribution of associative familiarity to recognition of unitized associations.

The current research builds upon recent studies that have used event related potentials (ERPs) to identify neural correlates of familiarity-based associative memory (Jager, Mecklinger, and Kipp, 2006; Opitz and Cornell, 2006), as well as prior functional MRI studies that have examined the contribution of distinct MTL subregions to memory performance. For example, Davachi and her colleagues have shown that perirhinal cortex codes the specific visual and conceptual features of objects, while the hippocampus forms domain-general relational representations. The current findings extend this research by demonstrating that engagement of perirhinal cortex depends not only on the type of information to be processed, but also the mnemonic demands of the task (i.e., familiarity for unitized associations).

The notion that perirhinal activity can support memory for unitized associations may explain why perirhinal cortex activity has been found to be correlated with successful associative recognition under conditions in which participants may encode stimuli in a unitized or gestalt-like manner (Yonelinas, 1999). Indeed, a consideration of these findings, as well as those from studies showing a correlation between perirhinal cortex activity and source recognition (e.g., background color of a studied word), has led to the proposal that perirhinal cortex may encode feature-fused item representations that can support later source or associative recognition judgments on the basis of familiarity (Diana et al., 2008; Haskins et al., 2009; Staresina and Davachi, 2006, 2008). However, perirhinal cortex activity has not been shown to correlate with source memory for contextual details (e.g., the task that was performed; Staresina and Davachi, 2008). Rather, retrieval of such non-unitized or flexible associations is mediated by the hippocampus (for a review see Eichenbaum, Yonelinas, 
Ranganath, 2007). The current findings provide further support for the notion that retrieval of unitized and non-unitized associations is mediated by perirhinal and hippocampal regions, respectively.

\section{Acknowledgments}

This work was supported by AG028774, MH57681, and the Medical Research Service of the Department of Veterans Affairs.

\section{References}

Aggleton JP, Vann SD, Denby C, Dix S, Mayes AR, Roberts N, Yonelinas AP. Sparing of the familiarity component of recognition memory in a patient with hippocampus pathology. Neuropsychologia 2005;43:1810-1823. [PubMed: 16154457]

Bowles B, Crupi C, Mirsattari S, Pigott S, Parrent A, Pruessner J, et al. Impaired familiarity with preserved recollection after anterior temporal-lobe resection that spares the hippocampus. Proceedings of the National Academy of Sciences of the United States of America 2007;104:16382-16387. [PubMed: 17905870]

Brainerd C, Reyna V. Fuzzy-trace theory and false memory. Current Directions in Psychological Science 2002;11:164-169.

Brainerd C, Wright R, Reyna V, Mojardin A. Conjoint recognition and phantom recollection. Journal of Experimental Psychology: Learning, Memory, and Cognition 2001;27:307-327.

Brett M, Anton J-L, Valabregue R, Poline J-B. Region of interest analysis using an SPM toolbox. NeuroImage 2002;16 abstract 497.

Daselaar SM, Fleck MS, Cabeza R. Triple dissociation in the medial temporal lobes: Recollection, familiarity, and novelty. Journal of Neurophysiology 2006;96:1902-11. [PubMed: 16738210]

Davachi L. Item, context and relational episodic encoding in humans. Current Opinion in Neurobiology 2006;16:693-700. [PubMed: 17097284]

Davachi L, Mitchell JP, Wagner AD. Multiple routes to memory: Distinct medial temporal lobe processes build item and source memories. PNAS 2003;100:2157-2162. [PubMed: 12578977]

Diana RA, Yonelinas AP, Ranganath C. Imaging recollection and familiarity in the medial temporal lobe: A three-component model. TRENDS in Cognitive Sciences 2007;11:379-386. [PubMed: 17707683]

Diana RA, Yonelinas AP, Ranganath C. The effects of unitization on familiarity-based source memory: Testing a behavioral prediction derived from neuroimaging data. Journal of Experimental Psychology: Learning, Memory, and Cognition 2008;34:730-740.

Eichenbaum H, Yonelinas AP, Ranganath C. The medial temporal lobe and recognition memory. Annual Review of Neuroscience 2007;30:123-152.

Fernández G, Tendolkar I. The rhinal cortex: 'Gatekeeper' of the declarative memory system. Trends in Cognitive Sciences 2006;10:359-362.

Giovanello KS, Keane MM, Verfaellie M. The contribution of familiarity to associative memory in amnesia. Neuropsychologia 2006;44:1859-1865. [PubMed: 16643967]

Giovanello KS, Schnyer DM, Verfaellie M. A critical role for the anterior hippocampus in relational memory: Evidence from an fMRI study comparing associative and item recognition. Hippocampus 2004;14:5-8. [PubMed: 15058477]

Giovanello KS, Schnyer D, Verfaellie M. Distinct hippocampal regions make unique contributions to relational memory. Hippocampus 2009;19:111-117. [PubMed: 18727049]

Giovanello KS, Verfaellie M, Keane MM. Disproportionate deficits in associative recognition relative to item recognition in global amnesia. Cognitive, Affective, and Behavioral Neuroscience 2003;3:186-194.

Haskins AL, Yonelinas AP, Quamme JR, Ranganath C. Perirhinal cortex supports encoding and familiarity-based recognition of novel associations. Neuron 2008;59:554-560. [PubMed: 18760692] 
Henke K, Buck A, Weber B, Wieser HG. Human hippocampus establishes associations in memory. Hippocampus 1997;7:249-256. [PubMed: 9228523]

Hockley W, Consoli A. Familiarity and recollection in item and associative recognition. Memory and Cognition 1999;27:657-664.

Jager T, Mecklinger A, Kipp K. Intra- and Inter-item Associations doubly dissociate the electrophysiological correlates of familiarity and recollection. Neuron 2006;52:535-545. [PubMed: 17088218]

Johnson JD, Rugg MD. Recollection and the reinstatement of encoding-related activity. Cerebral Cortex 2007;17:2507-2515. [PubMed: 17204822]

Jones T, Jacoby L. Feature and conjunction errors in recognition memory: Evidence for dual-process theory. Journal of Memory and Language 2001;45:82-102.

Jones T, Jacoby L, Gellis L. Cross-modal feature and conjunction errors in recognition memory. Journal of Memory and Language 2001;44:131-152.

Kirwan C, Stark C. Medial Temporal Lobe Activation During Encoding and Retrieval of Novel FaceName Pairs. Hippocampus 2004;14:919-930. [PubMed: 15382260]

Kirwan CB, Wixted JT, Squire LR. A demonstration that the hippocampus supports both recollection and familiarity. Proceedings of the National Academy of Sciences 2010;107:344-348.

Lampinen J, Odegard T, Neuschatz J. Robust Recollection Rejection in the Memory Conjunction Paradigm. Journal of Experimental Psychology: Learning, Memory, and Cognition 2004;30:332342.

Lieberman M, Cunningham WA. Type I and Type II error concerns in fMRI research: Re-balancing the scale. Social Cognitive and Affective Neuroscience 2009;4:423-428. [PubMed: 20035017]

Mandler G. Recognizing: The judgment of previous occurrence. Psychological Review 1980;87:252271.

Mayes AR, Montaldi D, Migo E. Associative memory and the medial temporal lobe. Trends in Cognitive Sciences 2007;11:126-135. [PubMed: 17270487]

Montaldi D, Spencer TJ, Roberts N, Mayes A,R. The neural system that mediates familiarity memory. Hippocampus 2006;16:504-20. [PubMed: 16634088]

Norman K, O'Reilly R. Modeling hippocampal and neocortical contributions to recognition memory: A complementary-learning-systems approach. Psychological Review 2003;110:611-646. [PubMed: 14599236]

Opitz B, Cornell. Contribution of familiarity and recollection to associative recognition memory: Insights from event-related potentials (ERP). Journal of Cognitive Neuroscience 2006;18:15951605. [PubMed: 16989559]

Quamme JR, Yonelinas AP, Norman KA. Effect of Unitization on Associative Recognition in Amnesia. Hippocampus 2007;17:192-200. [PubMed: 17203466]

Reinitz M, Morrissey J, Demb J. Role of attention in face encoding. Journal of Experimental Psychology: Learning, Memory, and Cognition 1994;20:161-168.

Rotello C, Heit E. Two-process models of recognition memory: Evidence for recall-to-reject? Journal of Memory and Language 1999;40:432-453.

Rotello C, Heit E. Associative recognition: A case of recall-to-reject processing. Memory \& Cognition 2000;28:907-922.

Rotello C, Macmillan N, Van Tassel G. Recall-to-reject in recognition: Evidence from ROC curves. Journal of Memory and Language 2000;43:67-88.

Staresina BP, Davachi L. Selective and shared contributions of the hippocampus and perirhinal cortex to episodic item and associative encoding. Journal of Cognitive Neuroscience 2008;20:1478-1489. [PubMed: 18303974]

Stark C, Squire L. Simple and associative recognition memory in the hippocampal region. Learning \& Memory 2001;8:190-197. [PubMed: 11533222]

Stark ELC, Bayley PJ, Squire LR. Recognition memory for single items and for associations is similarly impaired following damage to the hippocampal region. Learning and Memory 2002;9:238-242. [PubMed: 12359833] 
Stark ELC, Squire LR. Hippocampal damage equally impairs memory for single items and memory for conjunctions. Hippocampus 2003;13:281-292. [PubMed: 12699335]

Turriziani P, Fadda L, Caltagirone C, Carlesimo GA. Recognition memory for single items and for associations in amnesic patients. Neuropsychologia 2004;42:426-433. [PubMed: 14728917]

Turriziani P, Serra L, Fadda L, Caltagirone C, Carlesimo G. Recollection and familiarity in hippocampus amnesia. Hippocampus 2008;18:469-480. [PubMed: 18306303]

Wais P. Hippocampal Signals for strong memory when associative memory is available and when it is not. Hippocampus. in press.

Wais P, Squire L, Wixted J. In search of recollection and familiarity signals in the hippocampus. Journal of Cognitive Neuroscience 2010;22:109-123. [PubMed: 19199424]

Wais P, Wixted JT, Hopkins RO, Squire LR. The hippocampus supports both the recollection and the familiarity components of recognition memory. Neuron 2006;49:459-468. [PubMed: 16446148]

Wong M, Rotello C. Conjunction errors and semantic transparency. Memory \& Cognition 2010;38:47-56.

Yonelinas A. The contribution of recollection and familiarity to recognition and source-memory judgments: A formal dual-process model and an analysis of receiver operating characteristics. Journal of Experimental Psychology: Learning, Memory, and Cognition 1999;25:1415-1434.

Yonelinas AP, Kroll NEA, Dobbins IG, Soltani M. Recognition memory for faces: When familiarity supports associative recognition judgments. Psychonomic Bulletin \& Review 1999;6:654-661. [PubMed: 10682209]

Yonelinas AP, Hopfinger JB, Buonocore MH, Kroll NEA, Baynes K. Hippocampal, parahippocampal, and occipital-temporal contributions to associative and item recognition memory: An fMRI study. Neuroreport: For Rapid Communication of Neuroscience Research 2001;12:359-363. 


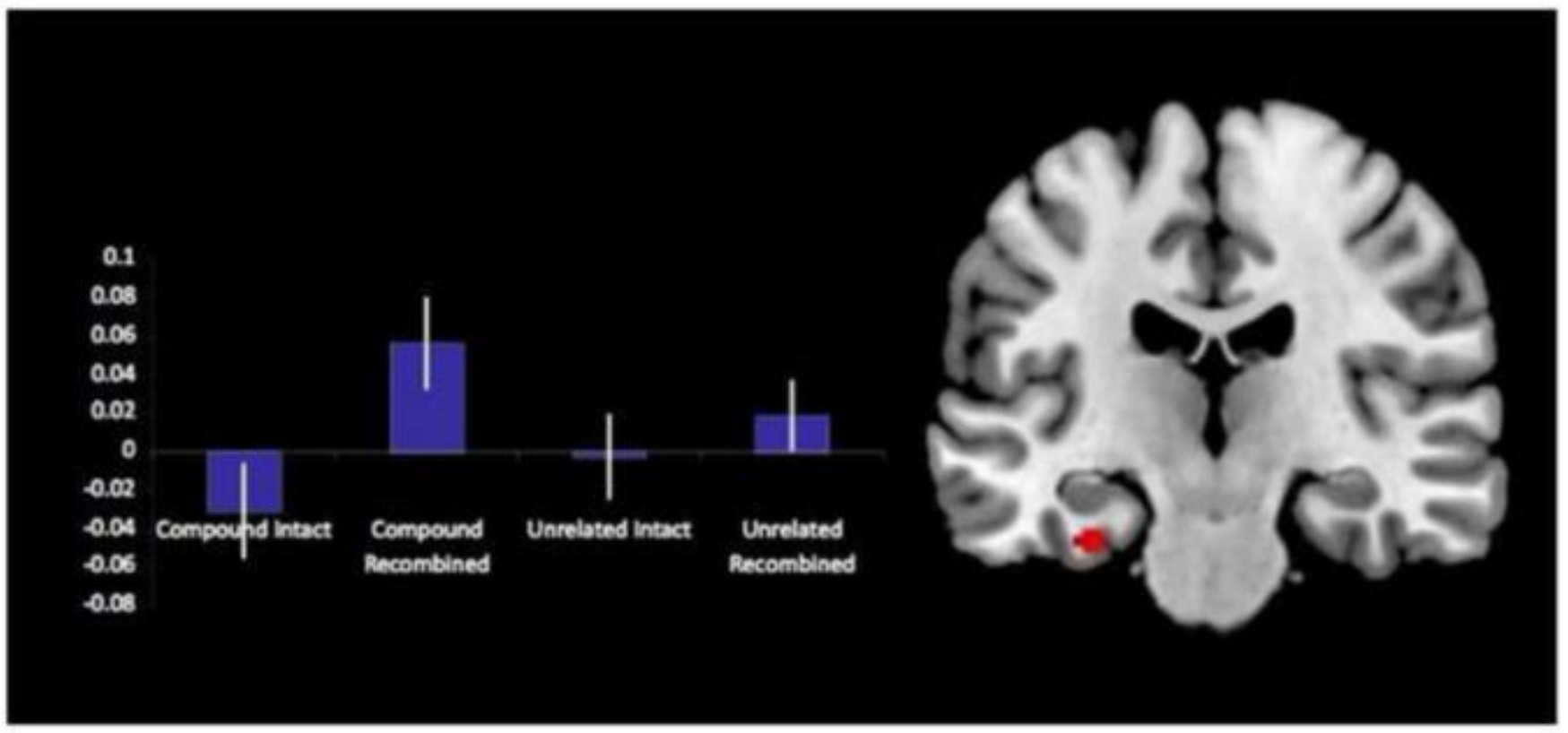

Figure 1.

Region of interest associated with compound words (intact > recombined), greater than unrelated word pairs (intact > recombined): Left BA35: $-30,-20,-26$. Graph depicts mean percent signal change associated with each memory condition (compound intact, compound recombined, unrelated intact, unrelated recombined) within the region of interest. 


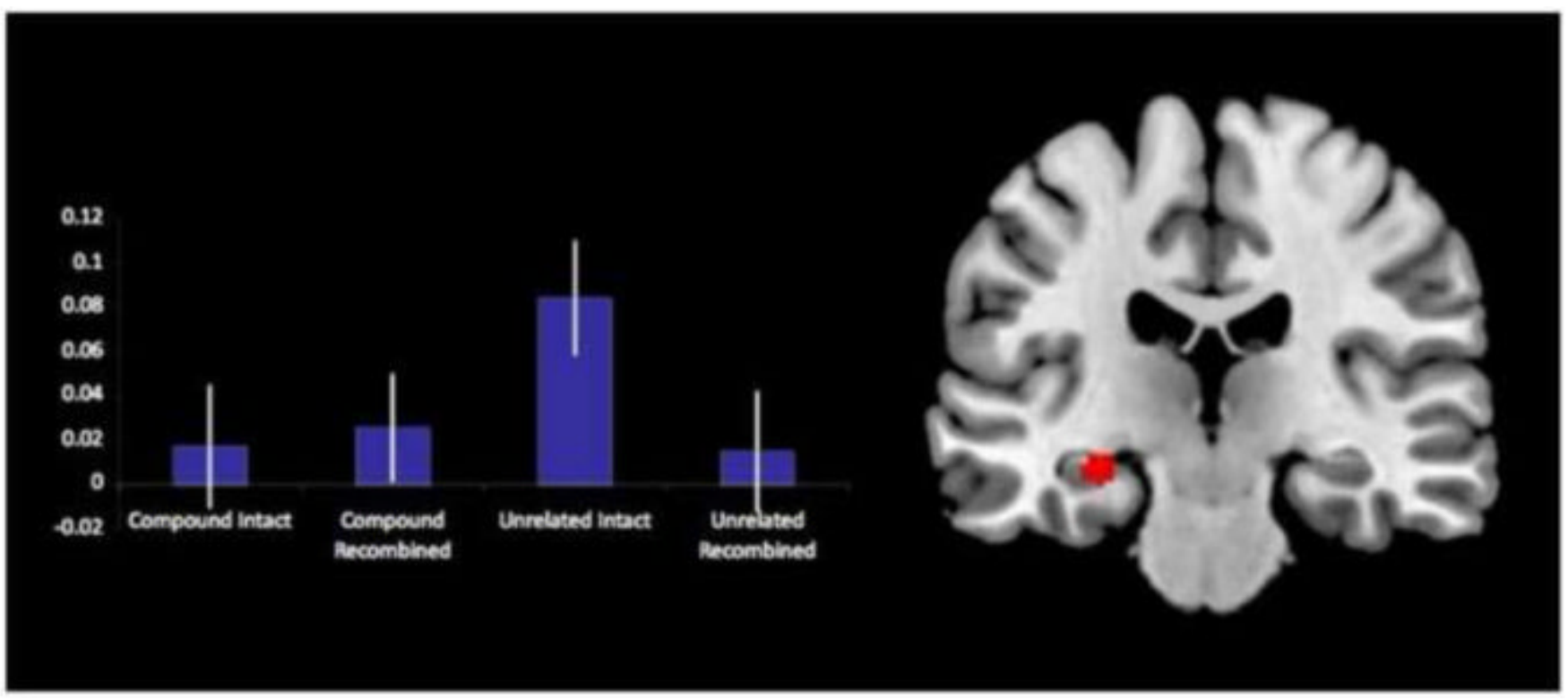

Figure 2.

Region of interest associated with unrelated word pairs (intact $>$ recombined), greater than compound word pairs (intact > recombined): Left Hippocampus: $-28,-22,-14$. Graphs depict mean percent signal change associated with each memory condition (compound intact, compound recombined, unrelated intact, unrelated recombined) within the region of interest. 


\section{Table 1}

Summary of behavioral data

\begin{tabular}{lrr}
\hline & Unrelated Word Pairs & Compound Words \\
Corrected Accuracy & $.62(.04)$ & $.60(.04)$ \\
$\quad$ Hits & $.77(.03)$ & $.81(.03)$ \\
$\quad$ False Alarms & $.15(.02)$ & $.21(.03)$ \\
Response Times (s) & & \\
$\quad$ Hits & $1.51(.04)$ & $1.27(.03)$ \\
$\quad$ Correct Rejections & $1.79(.05)$ & $1.45(.05)$ \\
Study Durations (s) & $4.4(.26)$ & $3.4(.28)$ \\
\hline
\end{tabular}

Note: Standard errors are presented in parentheses 


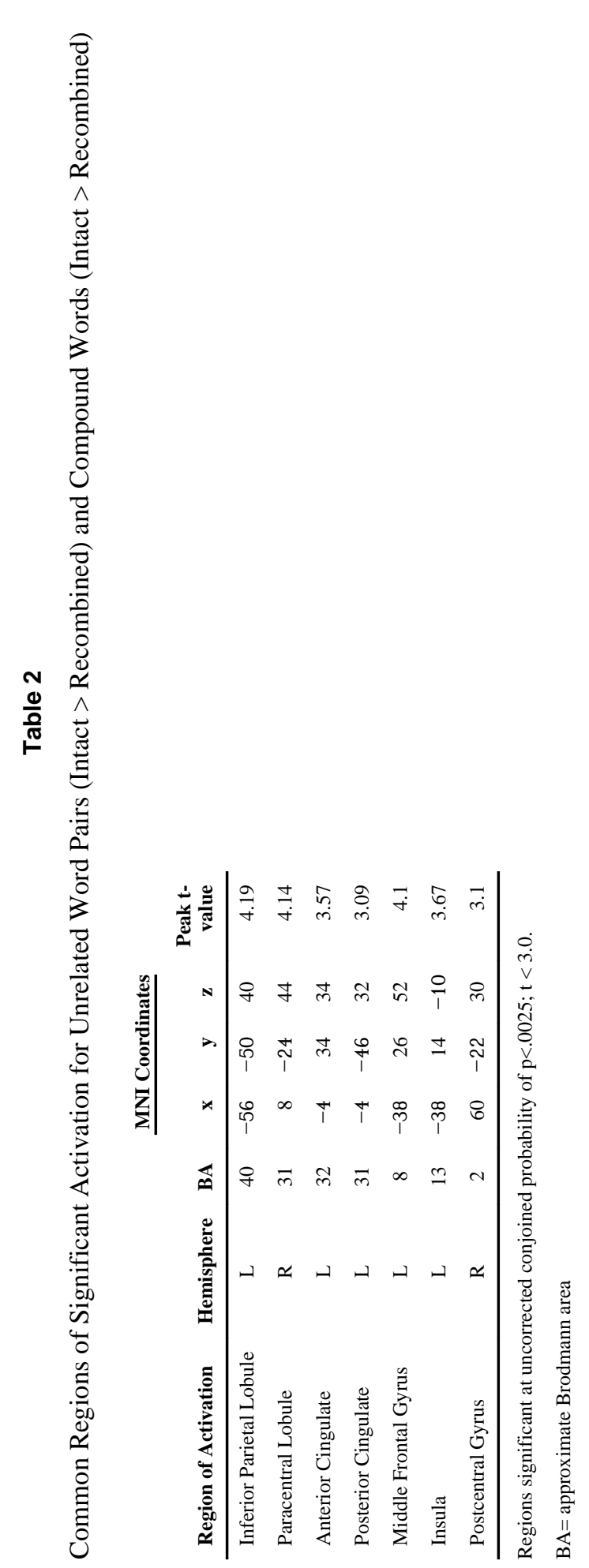

Neuropsychologia. Author manuscript; available in PMC 2011 August 1. 


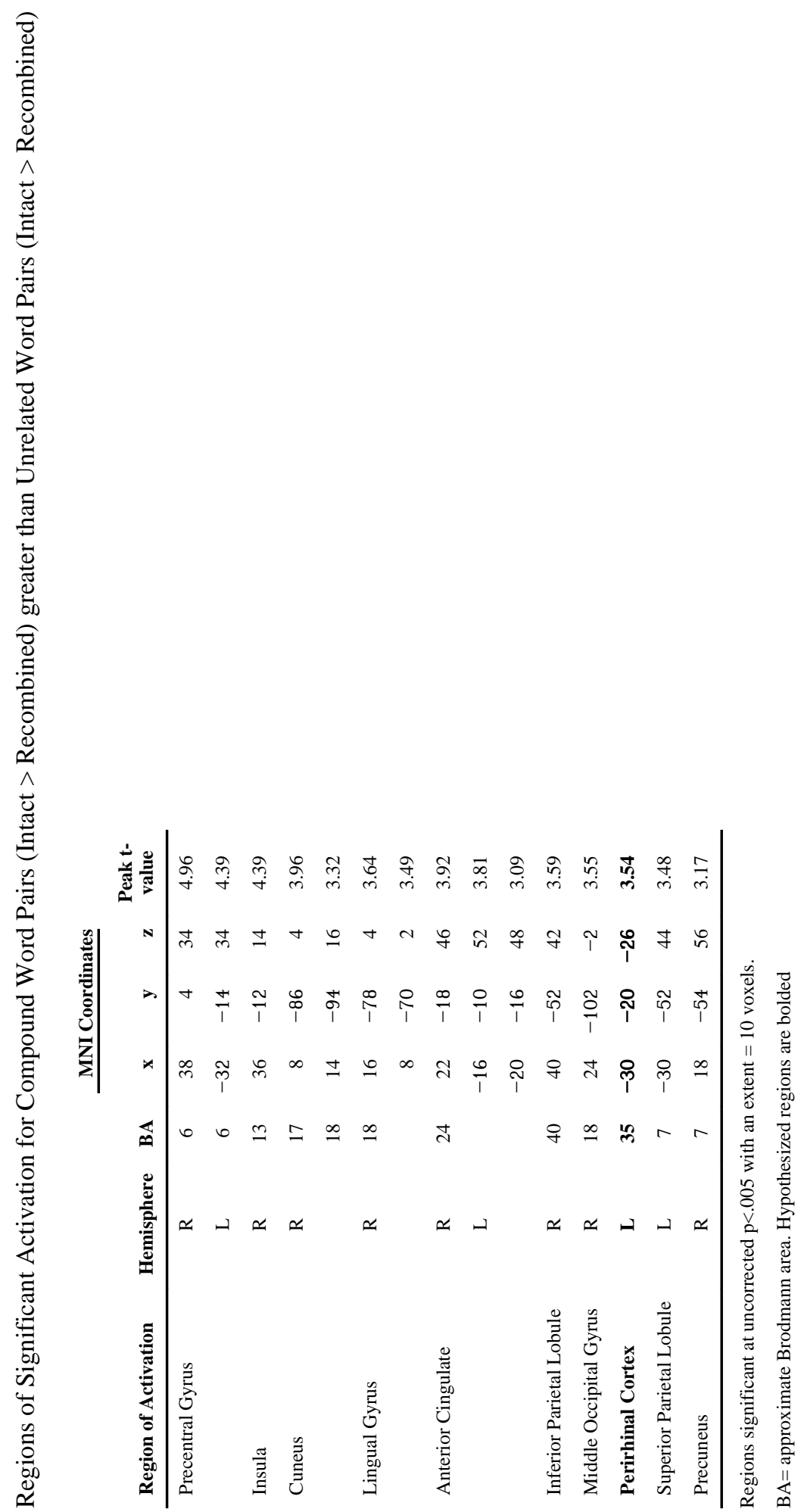

Neuropsychologia. Author manuscript; available in PMC 2011 August 1. 


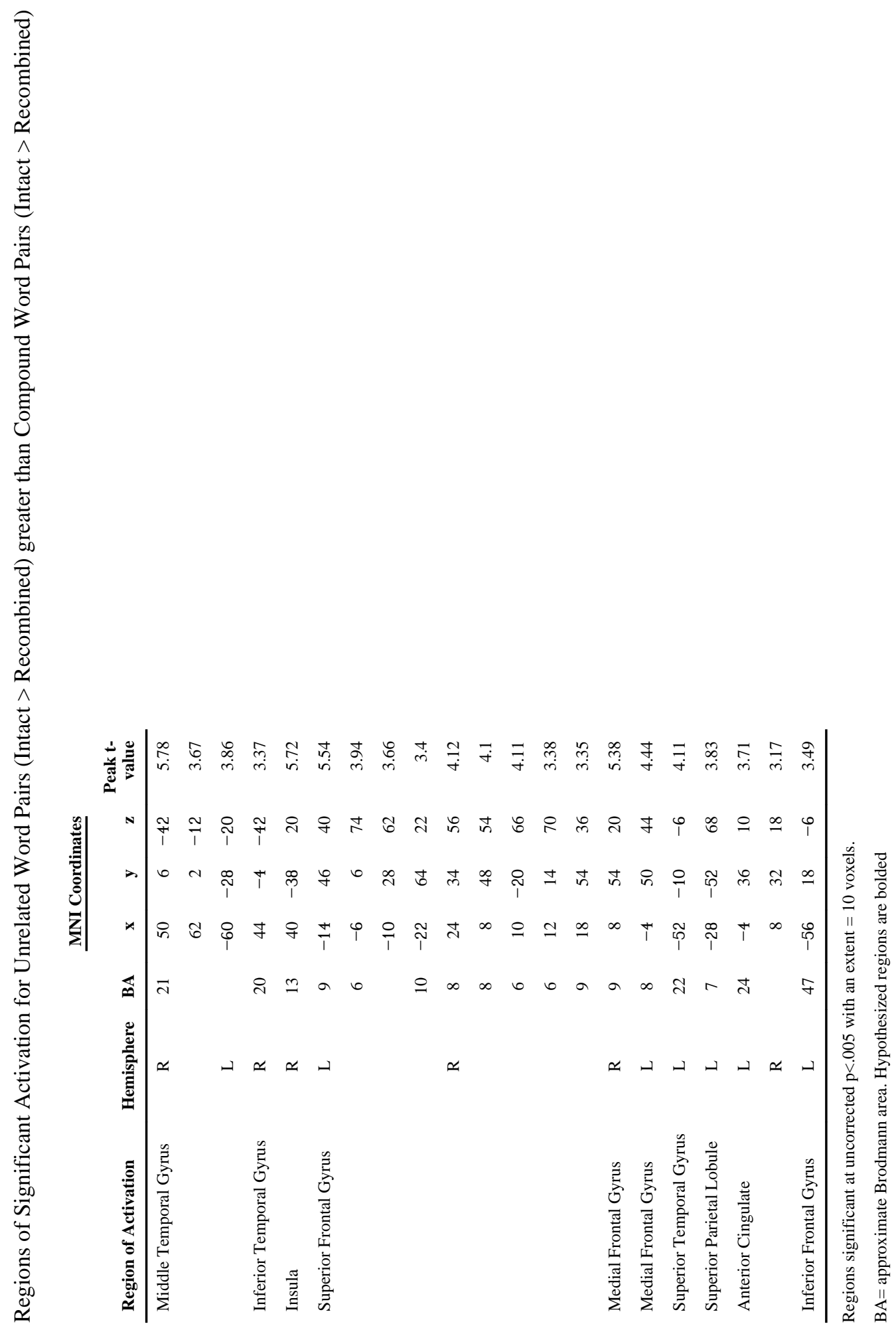

